to Minimize Crop Loss

PROCEEDINGS (c) 2003 Plant Management Network.

Accepted for publication 16 December 2002. Published 13 November 2003.

\section{The Maximum Pest Limit Concept Explained}

\author{
Gareth Hughes, School of Biological Sciences, University of \\ Edinburgh, Edinburgh EH9 3J G, UK
}

Corresponding author: Gareth Hughes. ghughes@srv0.bio.ed.ac.uk

Hughes, G. 2003. The maximum pest limit concept explained. Online. Plant Health Progress doi: 10.1094/PHP-2003-1113-05-RV.

\section{Abstract}

The maximum pest limit (MPL) concept was developed as a practical method of implementing quarantine security measures against the import of invasive pest species of plants. The MPL itself is simply a threshold upper limit, above which the pest species in question is deemed capable of establishing a population if imported in a consignment of fruit or vegetables. This limit depends on various biological and ecological characteristics of the pest species in question. Important aspects of implementation relate to how treatment and sampling may be combined to reduce the probability that the MPL will be exceeded. If a specified level of treatment efficacy is required (for example, probit nine level), then choice of an appropriate sample size becomes the main problem for regulatory authorities seeking to maintain quarantine security.

\section{I ntroduction}

This article is intended to provide a mostly non-mathematical account of the maximum pest limit concept. Readers who would prefer to work through the mathematical details from the outset are referred to the Literature Cited section, and the articles listed therein. Notwithstanding the intention to avoid presentation of most of the mathematical details of the maximum pest limit concept, some notation used in mathematical formulations of the concept will be retained, chiefly as a form of shorthand. Quantitative aspects of the concept will be illustrated graphically.

The ideas outlined in an article by Landolt et al. (5) underlie much of the subsequent work on maximum pest limits. Landolt et al. (5) pointed out that specification of a required level of treatment efficacy (usually 'probit nine', a survival rate of $32 \times 10^{-6}\left[=10^{-4.5}\right]$ ) did not guarantee quarantine security, because the probability of pest introduction depends on the number of pests surviving treatment, rather than the number of pests killed. Treatment obviously reduces this probability, but not in any quantifiable way unless we also have information on the pre-treatment level of infestation and other biological and ecological data (as discussed later in this article) on the pest species in question. In the context of measures taken against the introduction of fruit flies, Landolt et al. (5) suggested that the risk of pest introduction, calculated as the probability of a potential mating pair arriving per consignment, provided a basis for a more consistent approach to quarantine security.

\section{The Maximum Pest Limit}

The term 'maximum pest limit' (MPL) appears to have been introduced to the literature by Baker et al. (1), who were concerned to avoid the establishment of fruit flies in New Zealand through imported produce. They defined the maximum pest limit as the maximum number of fruit flies that can be present in consignments imported during a specified time to a specified location, such that the smallest number of flies capable of establishing a colony is not exceeded. Baker et al. (1) set this limit at three live larvae, arguing that natural mortality should ensure that this would not subsequently result in a mating pair.

Sampling to comply with an MPL, based on the cost to the exporter. Baker et al. (1) envisaged the following scenario. Consignments of fruit arrive from various sources (having been treated prior to shipping in order to qualify for a phytosanitary certificate) to be consolidated at a single destination in a specified time period (a single day is usually the time unit used for the calculations). The probability that the MPL is not exceeded after 
treatment in the consolidated consignment for any day is denoted $\operatorname{Pr}(\mathrm{B})$ and depends on the adopted MPL $(\mathrm{m})$, the total number of fruit $(\mathrm{N})$, the proportion of fruit infested ( $p$ ), the mean number of pests per infested fruit $(\mu)$ and the survival rate after treatment (s). If we adopt an appropriate statistical probability distribution for $\operatorname{Pr}(\mathrm{B})$ and have agreed values for $\mathrm{m}, \mathrm{N}, \mu$, and $\mathrm{s}$, we can plot $\operatorname{Pr}(\mathrm{B})$ as a function of $\mathrm{p} . \operatorname{Pr}(\mathrm{B})$ decreases with increasing $\mathrm{p}$ (Fig. 1). The consolidated consignment is sampled. The probability that the pest is detected in the sample is denoted 1-Pr(A) and depends on $\mathrm{p}$ and the sample size (n). With an appropriate statistical probability distribution for $\operatorname{Pr}(\mathrm{A})$ and a value for $\mathrm{n}$, we can plot 1-Pr(A) as a function of p. 1-Pr(A) increases with increasing p (Fig. 1).

Baker et al. (1) then outlined the calculation of required sample size as follows. An acceptable risk $(\alpha)$ that pests will be detected in a sample taken from a consignment that is actually below the MPL is identified. Baker et al. (1) adopted $\alpha=0.95$. Note that Baker et al. (1) used the term 'risk' to refer to the probability of occurrence of an unfavorable event, a usage retained throughout the present article. A critical value of $p\left(p^{*}\right)$ at which $\operatorname{Pr}(B)=x^{0.5}$ is obtained, and $\mathrm{n}$ is then selected so that $1-\operatorname{Pr}(\mathrm{A})=\mathrm{\alpha}^{0.5}$ when $\mathrm{p}=\mathrm{p}^{*}$. Thus, at the critical value $p^{*}, \operatorname{Pr}(B) \times[1-\operatorname{Pr}(A)]=\alpha$. The probability that the MPL is not exceeded (after treatment) in the consolidated consignment for any day and that the pest is detected in the sample taken from this consignment is equal to a specified value $\alpha$ when $p=p^{*}$ (Fig. 1). The implication appeared to be that $\alpha$ was the maximum risk that pests will be detected in a sample taken from a consignment that is actually below the MPL, but this is not necessarily the case. In a more recent article (4) an alternative method was given for finding the value of $n$ such that the maximum value of $\operatorname{Pr}(\mathrm{B}) \times[1-\operatorname{Pr}(\mathrm{A})]$ as a function of $\mathrm{p}$ was equal to $\alpha$.

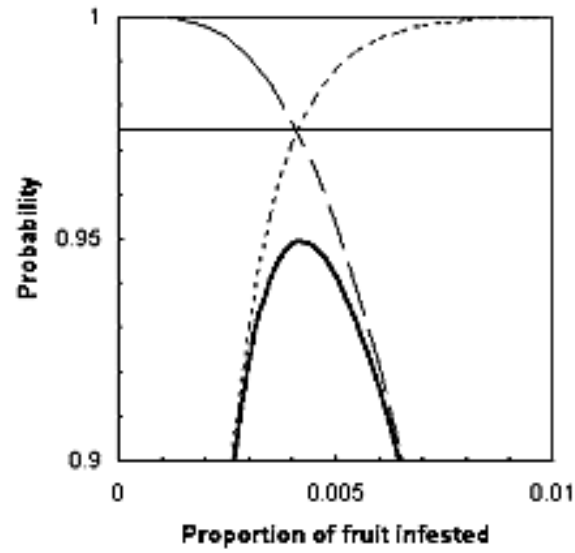

Fig. 1. Sampling to comply with a maximum pest limit, based on the cost to the exporter. The figure shows a graphical interpretation of equation 6 from Baker et al. (1), with $m=3$, $\mathrm{N}=208550, \mu=40, s=10^{-4.5}, \alpha=0.95$. The dashed line (- - ) shows the probability that the consolidated consignment is below the maximum pest limit after treatment, $\operatorname{Pr}(B)$, as a function of the proportion of fruit infested $p$. This line intersects with $\alpha^{0.5}$ (shown by a solid horizontal line) at $p^{*}=0.0041$, characterizing the critical incidence of infestation for the selected parameter values above. The dotted line (---) shows the probability that the pest is detected in the sample $1-\operatorname{Pr}(A)$ as a function of the proportion of fruit infested $p$, with $\operatorname{Pr}(A)=e^{-n p}$. The sample size $n$ is chosen so that $1-\operatorname{Pr}(A)$ as a function of $p$ intersects $\operatorname{Pr}(B)$ as a function of $p$ at the previously identified critical incidence of infestation. The solid bold line then shows the probability that the consolidated consignment is below the maximum pest limit after treatment and that the pest is detected in the sample $\operatorname{Pr}(B) \times[1$ $\operatorname{Pr}(\mathrm{A})]$ as a function of $\mathrm{p}$. This probability has a value equal to $\alpha$ at the critical incidence of infestation.

The approach to sample size calculation adopted by Baker et al. (1) and Harte et al. (4) is based on setting a maximum value $\alpha$ for the risk that pests will be detected in a sample taken from a consolidated consignment in which the pest population is actually at or below the MPL. If detection of pests in a sample taken from a consolidated consignment results in a decision not to import, this would be an erroneous decision in cases in which the pest population in a consignment was actually at or below the MPL. Such decisions represent costs to the exporter(s). Presumably, a high value of $\alpha$ was adopted because this means that the corresponding risk of a decision, based on sampling, to import a 
consignment that is actually above the MPL will be low. The risk of a decision, based on sampling, to import a consignment that is actually above the MPL is not directly specified in the calculations described by Baker et al. (1), but it can be calculated retrospectively.

Following on from the work of Baker et al. (1), Mangan et al. (6) adopted the same statistical probability distribution for $\operatorname{Pr}(B)$ and with a specified value of $m$ and values of $N, \mu$, and $p$ derived from field data, defined $\operatorname{Pr}(\mathrm{B})$ (the probability that the MPL is not exceeded after treatment) as a function of survival rate after treatment (s). Pr(B) decreases with increasing s (Fig. 2). Mangan et al. (6) were interested in the critical value of $s\left(s^{*}\right)$ at which $\operatorname{Pr}(B)=\alpha^{0.5}$. If this value were less than $10^{-4.5}$, the implication would be that the survival rate after treatment required to comply with the adopted MPL was lower than probit nine level. Thus, if the actual survival rate after treatment was (or was assumed to be) probit nine level, additional pest control measures designed to reduce the pretreatment level of infestation would be required in order to comply with the adopted MPL.

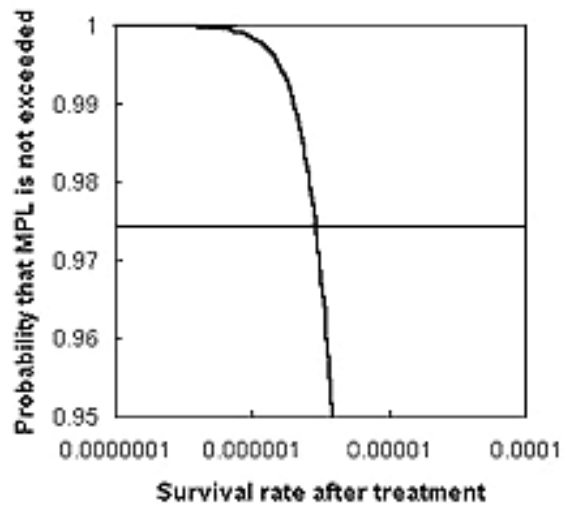

Fig. 2. A graphical interpretation of equation 6 from Baker et al. (1), with $m=2$, $\mathrm{N}=141250, \mu=7.427, p=0.205, \alpha=0.95$ (data from Mangan et al. (6)). The solid bold line shows the probability that the consignment is below the maximum pest limit after treatment $\operatorname{Pr}(\mathrm{B})$ as a function of the survival rate after treatment $\mathrm{s}$. For the selected parameter values above, this line intersects with $\alpha^{0.5}$ (shown by a solid horizontal line) between $s=10^{-5}$ and $s=10^{-6}$, indicating that the survival rate after treatment required to meet the adopted maximum pest limit was lower than probit nine level, $s=10^{-4.5}$. Thus, if the actual survival rate after treatment was probit nine level, additional pest control measures designed to reduce the pre-treatment level of infestation would be required in order to comply with the adopted maximum pest limit.

\section{Sampling to comply with an MPL, based on the risk to the} importer. The scenario envisaged by Cannon (2) differs from that outlined in the work of Baker et al. (1), in that it deals with treatment and sampling carried out on consignments of fruit at source, prior to shipping (which does not preclude further sampling of a consignment at the destination). Cannon's (2) calculation of sample size is based on setting a maximum value ( $\beta)$, chosen according to the severity of the consequences, for the risk that pests will not be detected in a sample taken from a consignment in which the pest population is actually above the MPL. Cannon (2) adopted $\beta=0.05$. It is assumed that if any pests are detected in a sample, the consignment is rejected. The parameters required for this analysis are the same as outlined for the analysis of Baker et al. (1), with the addition of the probability that a pest that survives treatment does not die from natural causes during shipping (h), which will depend on the length of the journey. Now, however, the calculation of sample size (n) is such that the probability that the MPL is exceeded (after treatment) in the consignment to be shipped and that the pest is not detected in the sample taken from this consignment $[1-\operatorname{Pr}(\mathrm{B})] \times \operatorname{Pr}(\mathrm{A})$ (as a function of $\mathrm{p}$, Fig. 3) has a maximum value equal to $\beta$. If non-detection of pests in a sample taken from a consignment results in a decision to import, this would be an erroneous decision in cases in which the pest population in the consignment was actually above the MPL. Such decisions represent risks to the importer(s). A low maximum value for this risk is directly specified. The corresponding risk of a decision, based on sampling, to reject a consignment that is actually at or below the MPL can be calculated retrospectively. 


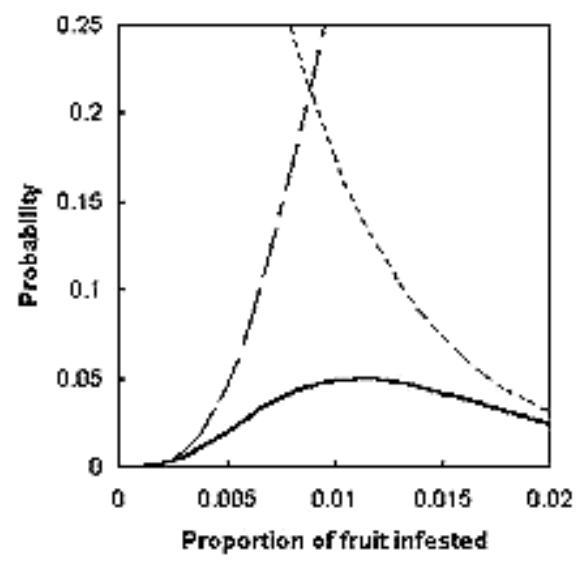

Fig. 3. Sampling to comply with a maximum pest limit, based on the risk to the importer. The dashed line (- - ) shows the probability that a consignment is above the maximum pest limit after treatment $1-\operatorname{Pr}(B)$ as a function of the proportion of fruit infested $p$, with:

$\operatorname{Pr}(B)=\sum_{r=0}^{m} \frac{q^{r}}{r !} \cdot e^{-q}$

and $\mathrm{q}=\mathrm{N} \times \mu \times \mathrm{s} \times \mathrm{h} \times \mathrm{p} ; \mathrm{m}=3, \mathrm{~N}=208550, \mu=40, \mathrm{~s}=10^{-4.5}, \mathrm{~h}=1$, data from Cannon (2). The dotted line (---) shows the probability that the pest is not detected in the sample $\operatorname{Pr}(A)$ as a function of the proportion of fruit infested $p$, with $\operatorname{Pr}(A)=e^{-n p}$. The sample size $n$ is chosen so that probability that a consignment is above the maximum pest limit after treatment and that the pest is not detected in the sample [1- $\operatorname{Pr}(B)] \times \operatorname{Pr}(A)$ (shown as a solid bold line) has a maximum value of $\beta$, with $\beta=0.05$ in this case.

Cannon (2) points out the importance of risk assessment based on the probability of establishment of a pest, not simply the probability of introduction. The same point is made by Yamamura and Katsumata (7), who discuss the probability of introduction as a component of quantitative risk assessment for quarantine pests.

\section{The Probability of I ntroduction}

The work of Yamamura and Katsumata (7) represents a return to the approach of Landolt et al. (5). Instead of adopting a threshold MPL and calculating the risk to importers or exporters of erroneous decisions about consignments in relation to that MPL, the probability that one or more reproductive individuals from a consignment of imported fruit pass the port is calculated directly. This probability, referred to as the probability of introduction $\operatorname{Pr}(\mathrm{I})$, depends on two characteristics of pests: the mode of reproduction (characterized as either parthenogenic or sexual) and pattern of infestation (characterized as either gregarious or solitary). Yamamura and Katsumata (7) formulated equations that allowed them to calculate $\operatorname{Pr}(I)$ for each of the four categories of pests: sexual, gregarious; sexual, solitary; parthenogenic, gregarious; and parthenogenic, solitary. The analysis for sexual, solitary pests corresponds to the analysis of Landolt et al. (5). Pr(I) depends on the total number of fruit $(\mathrm{N})$, the probability distribution of the proportion of fruit infested (parameters a and b), the probability distribution of live larvae per infested fruit (parameter v), the probability that an individual pest is female (f), the sample size (n) and the survival rate after treatment (s).

For each category of pests, Yamamura and Katsumata (7) derived equations that enabled prediction of the effects of two pest prevention practices before export: disinfestation treatment and subsequent sampling inspection. Using data from Mangan et al. (6) relating to the Mexican fruit fly (Anastrepha ludens) (classified as sexual, gregarious) to estimate the required parameters, Yamamura and Katsumata (7) characterized the probability of introduction per consignment as a function of survival rate after treatment (s), in the absence of sampling inspection. $\operatorname{Pr}(\mathrm{I})$ increases with increasing s (Fig. 4). 


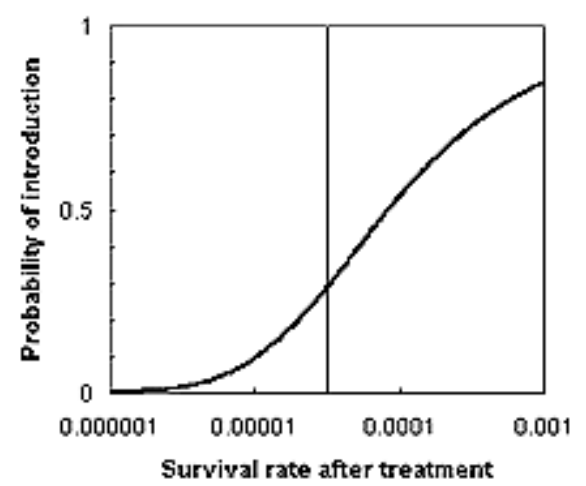

Fig. 4. A graphical interpretation of equation of equation 21 from Yamamura and Katsumata (7). The solid bold line shows the probability of introduction $\operatorname{Pr}(I)$ as a function of the survival rate after treatment $\mathrm{s}$. The parameters of the probability distribution of the proportion of fruit infested are $a=0.523$ and $b=5.42$, the parameter of the probability distribution of live larvae per infested fruit is $v=0.924$ (values calculated from data of Mangan et al., (6)), and the probability that an individual pest is female is $f=0.5$. Other selected parameter values are $\mathrm{N}=100000, \mathrm{n}=0$. For these parameter values, $\operatorname{Pr}(\mathrm{I})$ is approximately equal to 0.3 when the survival rate after treatment is probit nine level, $s=10^{-4.5}$, indicated by a solid vertical line.

From Fig. 4, we can see that with probit nine treatment efficacy (a survival rate of $\left.10^{-4.5}\right), \operatorname{Pr}(\mathrm{I})$ is approximately equal to 0.3 in the absence of sampling inspection $(n=0)$ for the selected parameter values derived from Mangan et al. (6). If this $\operatorname{Pr}(\mathrm{I})$ were regarded as too high, sampling could be carried out. As before, it is assumed that if any pests are detected in a sample, the consignment is rejected. Generally, $\operatorname{Pr}(\mathrm{I})$ is a decreasing function of sample size (Fig. 5). However, the efficiency of sampling is not independent of the survival rate after treatment. $\operatorname{Pr}(\mathrm{I})$ decreases more rapidly with increasing sampling rate when the survival rate after treatment is relatively high.

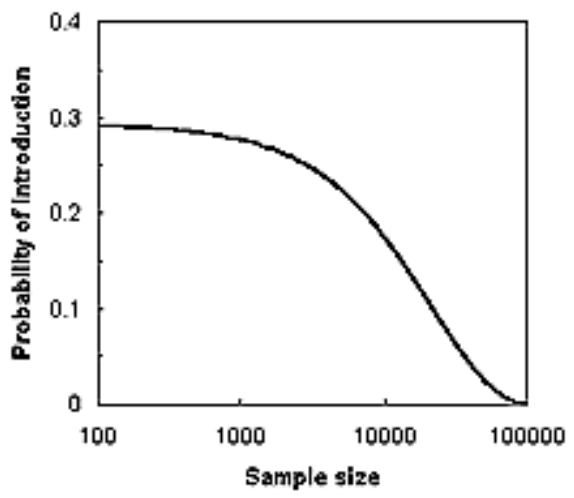

Fig. 5. A graphical interpretation of equation of equation 21 from Yamamura and Katsumata (7). The solid bold line shows the probability of introduction $\operatorname{Pr}(1)$ as a function of the sample size $n$. The parameters of the probability distribution of the proportion of fruit infested are $a=0.523$ and $b=5.42$, the parameter of the probability distribution of live larvae per infested fruit is $v=0.924$ (values calculated from data of Mangan et al., (6)), and the probability that an individual pest is female is $\mathrm{f}=0.5$ (all as in Fig. 4). Other selected parameter values are $\mathrm{N}=100000, \mathrm{~s}=10^{-4.5}$. For these parameter values, posttreatment sampling at a level of $20 \%$ of the consignment (i.e., 20,000 out of 100,000 fruits) brings about a reduction in the probability of introduction $\operatorname{Pr}(I)$ to approximately 0.1 .

\section{Conclusions}

The need for quantitative risk assessments for quarantine pests that meet the requirements for both reduction in the risk of invasion and transparency for the purpose of international trade has given rise to the concepts of the maximum pest limit and the probability of introduction. The MPL is a threshold, representing an upper limit to the number of pests, above which the pest species in question is deemed capable of establishing a population if imported in a consignment of fruit or vegetables. Treatment and sampling may be combined to reduce the probability that an adopted MPL will be exceeded. In the early work by Baker and colleagues $(1,4)$ the calculation of post-treatment sample size 
required specification of a value for the probability that pests will be detected in a sample taken from a consolidated consignment in which the pest population is actually at or below the MPL. Thus the operational implementation of the MPL was defined in terms of the cost to exporters of erroneously rejected consignments. More recent work by Cannon (2) based the calculation of posttreatment sample sizes on specification of a value for the probability that pests will not be detected in samples taken from consignments that are actually above the MPL. In this case, the operational implementation of the MPL was defined in terms of the risk to importers of erroneously accepted consignments. The latter probably corresponds more closely to the objective that regulatory authorities are trying to achieve in their risk assessments for quarantine pests. Note, however, that it is not a requirement that an acceptance sampling plan be designed exclusively from the point of view of either the exporter (producer) or the importer (consumer) (3).

As an alternative to the MPL approach, it is possible to calculate directly the probability of introduction $(4,7)$. In practice, this approach would first involve adoption of an appropriately low level for the acceptable probability of introduction, given the threat posed by a particular pest and the survival rate after treatment. The equations devised by Yamamura and Katsumata (7) would then be used to calculate the rate of post-treatment sampling required to reduce the per consignment probability of introduction to that level.

Readers should note that the preceding account represents only a descriptive outline of the articles mentioned, most of which are rather mathematical in their presentation. The papers by Cannon (2) and Yamamura and Katsumata (7), in particular, contain far more detail than it has been possible to summarize here and both merit further study.

\section{Literature Cited}

1. Baker, R. T., Cowley, J . M., Harte, D. S., and Frampton, E. R. 1990. Development of a maximum pest limit for fruit flies (Diptera: Tephritidae) in produce imported into New Zealand. J . Econ. Entomol. 83:13-17.

2. Cannon, R. M. 1998. Sampling to comply with a maximum pest limit. Biometrics 54:847-858.

3. Hald, A. 1967. The determination of single sampling attribute plans with given producer's and consumer's risk. Technometrics 9:401-415.

4. Harte, D. S., Baker, R. T., and Cowley, J. M. 1992. Relationship between preentry sample size for quarantine security and variability of estimates of fruit fly (Diptera: Tephritidae) disinfestation treatment efficacy. J . Econ. Entomol. 85:1560-1565.

5. Landolt, P. J., Chambers, D. L., and Chew, V. 1984. Alternative to the use of probit 9 mortality for quarantine treatments of fruit fly (Diptera: Tephritidae)-infested fruit. J. Econ. Entomol. 77:285-287.

6. Mangan, R. L., Frampton, E. R., Thomas, D. B., and Moreno, D. S. 1997. Application of the maximum pest limit concept to quarantine security standards for the Mexican fruit fly (Diptera: Tephritidae). J. Econ. Entomol. 90:1433-1440.

7. Yamamura, K., and Katsumata, H. 1999. Estimation of the probability of insect pest introduction through imported quantities. Res. Popul. Ecol. 41:275-282. 